\title{
Solid Immersion Lens Microscope for Spectroscopy of Nanostructure Materials
}

\author{
Sang-Youp Yim, Joon Heon Kim, and Jongmin Lee* \\ Advanced Photonics Research Institute, GIST, Gwangju 500-712, Korea
}

(Received November 4, 2010 : revised January 5, 2011 : accepted January 5, 2011)

\begin{abstract}
We demonstrate a high-spatial-resolution imaging and spectroscopy tool using a solid immersion lens (SIL), a hemispherical lens made of high refractive index glass $(n \sim 2)$. Photoluminescence (PL) images of single CdSe nanocrystals confirm a numerical aperture enhancement factor of $\sim 2$, close to the refractive index of the SIL. In particular, a bare-eye observation of PL signals emitted by single nanocrystals with $\sim 10 \mu \mathrm{m}^{-2}$ densities was possible over an $\sim 30 \mu \mathrm{m}$ diameter region. In addition, the PL spectra of single CdSe nanocrystals were successfully measured at room temperature. Thus, this SIL microscope ensures a simple but powerful method for nanostructure spectroscopy.
\end{abstract}

Keywords : Solid immersion lens, Microscopy, Spectroscopy, Nanostructure

OCIS codes : (180.2520) Fluorescence microscopy; (300.6280) Spectroscopy, fluorescence and luminescence; (160.4236) Nanomaterials

\section{INTRODUCTION}

High-spatial-resolution imaging has been demonstrated by reducing or even breaking the diffraction limit, using microscopic techniques, such as confocal laser scanning microscopy (CLSM) [1], near-field scanning optical microscopy (NSOM) [2], and stimulated emission depletion (STED) microscopy [3], among others. Solid immersion lens microscopy is one method used to enhance spatial resolution by "immersing" the sample in a solid material of a certain refractive index (n), referred to as a solid immersion lens (SIL) [4]. Although the spatial resolution of SIL microscopy does not reach that of NSOM or STED, it is still an attractive technique. First, an expensive microscope objective lens with a high numerical aperture (NA) is not necessary. In addition, the setup is straightforward, compared to the complicated and delicate setup of novel techniques like NSOM and STED. Moreover, high-resolution bare-eye observation is possible without requiring any additional instruments; commonly, realtime imaging using CLSM requires a high-speed scanner [1].

In general, SILs are in the form of either a hemisphere [5] or a super-hemisphere truncated at $r / n$ from the center ( $r$ is the radius) [6]. The NA enhancement factor is $n$ for the hemispherical SIL because the SIL simply replaces the air; incident rays from an objective lens along radii of the
SIL can converge to a spot on the flat surface of the SIL without changing the beam path. For the super-hemispherical SIL, an oblique incident ray can be focused perfectly to a spot at $r / n$ from the center. Here, the initial NA of an objective lens further increases $n$ times due to refraction at the rounded surface. Therefore NA enhancement factor is $n^{2}$ for the super-hemispherical SIL, though the maximum effective NA is $n$ for both types [4]. Therefore, within the limit of the maximum effective NA, the latter has a higher NA with the same objective lens. However, because the dimensions are dependent on $n$, the super-hemispherical SIL cannot avoid severe chromatic aberration, a key obstacle for broadband spectroscopy [7].

In this paper, we demonstrate a high-spatial-resolution imaging and spectroscopy tool using a hemispherical SIL. We take fluorescence signals from single CdSe nanocrystals as point sources to measure the instrument response. Based on subsequent measurements, our results confirm that the NA enhancement factor is close to the refractive index of the SIL, as expected. In particular, the bare-eye observation of PL signals emitted by single CdSe nanocrystals becomes possible, in which the PL spectra are successfully measured at room temperature.

\footnotetext{
*Corresponding author: leejm@gist.ac.kr

Color versions of one or more of the figures in this paper are available online.
} 


\section{SIL MICROSCOPE AND EXPERIMENTAL SETUP}

A SIL microscope was built on an inverted optical microscope (Ti-U, Nikon, Japan). For the SIL, we used a 2-mm-diameter hemisphere made of S-LAH79 glass (Ohara) having a refractive index of 2.00330 at the helium d-line $(587.5618$ $\mathrm{nm})$. Note that a refractive index of $\sim 2$ is one of the highest values of commercially available glass materials. Moreover, its absorption coefficient at $400 \mathrm{~nm}$ is as low as $0.693 \mathrm{~cm}^{-1}$, ensuring UV-visible spectroscopic applications.

A schematic of the experimental setup is shown in Fig. 1. We used a femtosecond Ti:sapphire oscillator system (MaiTai, Spectra Physics, USA) as the light source. The beam was converted to a second harmonic beam $(400 \mathrm{~nm}$ wavelength) using a $3-\mathrm{BaB}_{2} \mathrm{O}_{4}(\mathrm{BBO})$ crystal, and then filtered through a pinhole to make a smooth Gaussian profile. The beam was defocused so that the beam width might be large enough to provide the nearly uniform illumination of the sample plane over a $100 \mu \mathrm{m}$ spot; we did not measure the actual beam width at the sample plane, but confirmed that the intensity variation was negligible. Subsequently, the beam was guided to the optical microscope, reflected by a dichroic mirror designed for a $400 \mathrm{~nm}$ wavelength, and then fed to an objective lens $(0.6 \mathrm{NA}, 40 \times$ magnification power) before finally reaching the SIL. Then the beam excited the samples on top of the SIL; for the parallel experiments without the SIL, the samples were coated on a glass substrate and placed facing down to the objective lens to minimize aberrations. Fluorescence signals, collected by the same SIL and objective lens, were separated from the laser light using the dichroic mirror, and then guided to the detection ports.

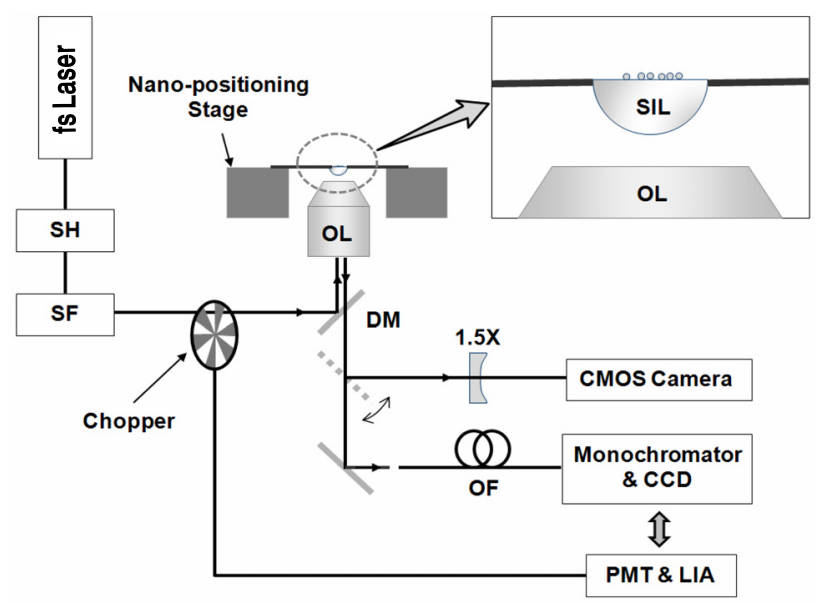

FIG. 1. Schematic of a SIL microscope setup combined with spectroscopy instruments. CCD: charge-coupled device, CMOS: complementary metal-oxide semiconductor, DM: dichroic mirror, LIA: lock-in amplifier, OF: optical fiber, OL: objective lens, PMT: photomultiplier tube, SH: second harmonic unit, SF: spatial filter unit, and SIL: solid immersion lens.
As mentioned above, a SIL microscope offers a high lateral resolution - even in a wide-field detection scheme. For comparison, we built a system that works in both wide-field and scanning modes, modes that can easily be switched by flipping a mirror on the microscope. A complementary metal-oxide semiconductor (CMOS) camera attached at one port was used to take images for a 111 $\mu \mathrm{m} \times 89 \mu \mathrm{m}$ view-field without a SIL, and a $51 \mu \mathrm{m} \times 41$ $\mu \mathrm{m}$ view-field with a SIL in wide-field mode. Before taken, the image was further magnified $1.5 \times$ to increase the measurement accuracy [7]; the camera was calibrated using a $4-\mu \mathrm{m}$-period calibration grating for both with and without the SIL.

For the pinhole in scanning mode, we used a 633-nm single-mode fiber, in which the core diameter was much smaller than a magnified Airy unit (1.22 $\lambda M / N A)$, where $\mathrm{M}$ denotes the magnification of the system. For detection, the collected signal from one point was measured by either a lock-in amplifier (LIA) combined with a photomultiplier tube (PMT), or by a Peltier cooled charge-coupled device (CCD) with a $32-\mathrm{cm}$ monochromator. Then, to construct an image, we scanned the sample rather than the beam. In general, the beam scanning method takes a shorter time for image acquisition. However, when a long data acquisition time is necessary for weak fluorescence signals, the sample scanning method is more stable since the sample can stay within a few $\mathrm{nm}$ as it has a closed loop nano-positioning stage. In this study, we used a nano-positioning stage (nPoint Inc., USA) having a position noise of less than 0.5 $\mathrm{nm}$, and hysteresis and linearity errors of less than $0.05 \%$ during closed loop operation. Automation of the scanning and data acquisition was performed with an execution code written in a LabVIEW environment.

\section{RESULTS AND DISCUSSION}

The SIL microscope successfully demonstrated the highspatial-resolution PL imaging of chemically synthesized single CdSe nanocrystals, which were directly coated on the flat surface of the SIL to minimize gap errors (Fig. 2). We could observe the fluorescence intermittency (blinking) effect of single CdSe nanocrystals [8] having $\sim 10 \mu \mathrm{m}^{-2}$ densities with our bare eyes. Note that this bare-eye observation was possible for diameters of over $\sim 30 \mu \mathrm{m}$ in aberration-free regions in wide-field mode.

Since the diameter of CdSe nanocrystals $(\leq 10 \mathrm{~nm})$ was much smaller than the lateral resolution of the system, the nanocrystals can be assumed to be point sources. Therefore, the intensity pattern of the diffraction-limited spot is the square of the amplitude point spread function (PSF) of the imaging optics, which takes the form of the Airy disc function [9]

$$
I(x)=I_{0}\left(\frac{2 J_{1}(x)}{x}\right)^{2} .
$$




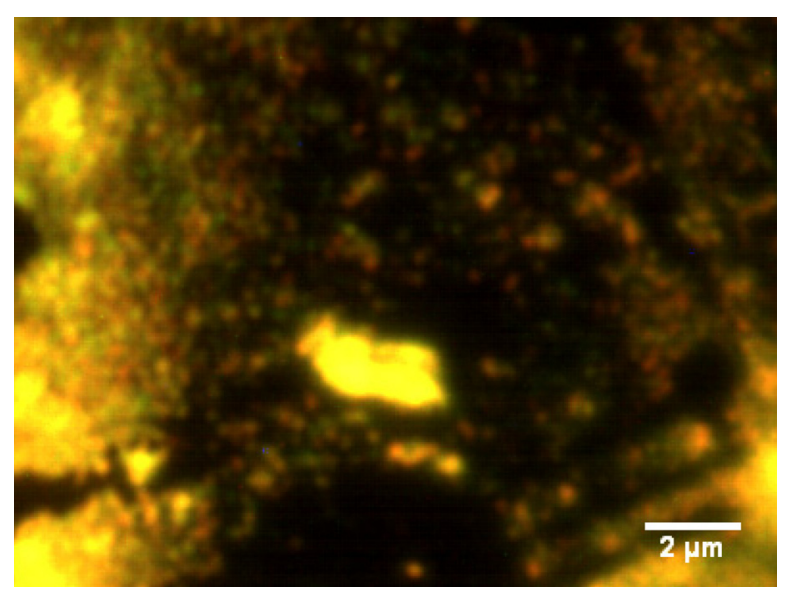

FIG. 2. Wide-field mode PL image of CdSe nanocrystals taken by the SIL microscope.
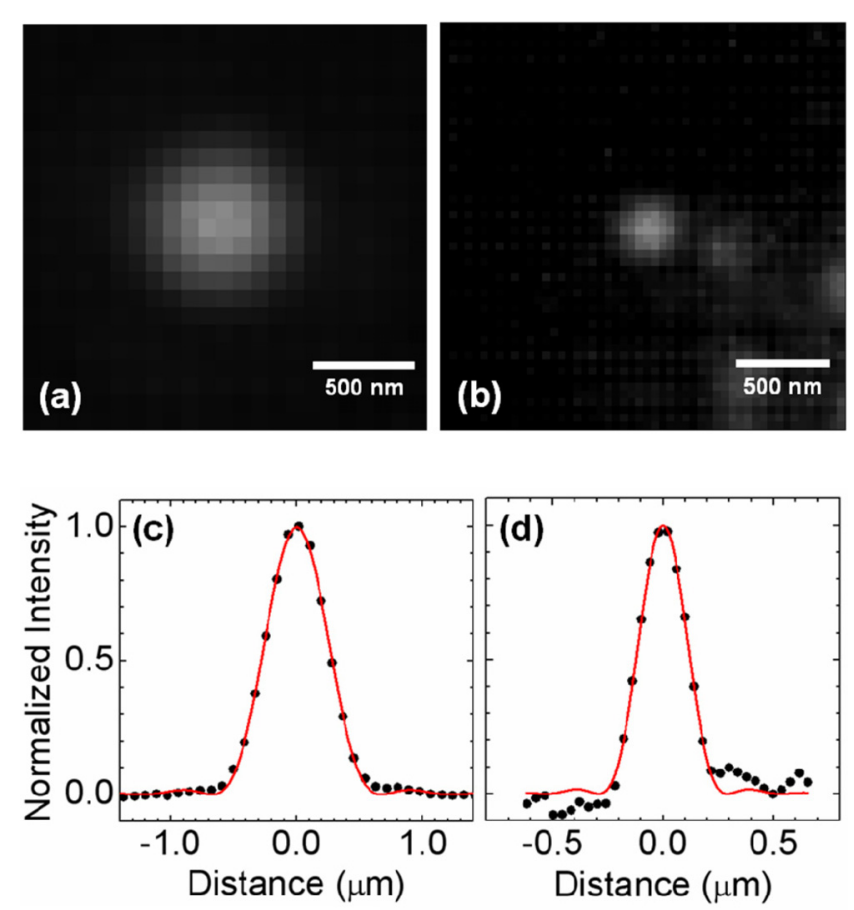

FIG. 3. Wide-field mode PL images and line profiles of single CdSe nanocrystals: (a) and (c) taken by a conventional optical microscope, and (b) and (d) taken by a SIL microscope. Measured PL spectra are at $590 \mathrm{~nm}$ and $581 \mathrm{~nm}$ peaks for the single nanocrystals of (a) and (b).

Here, $J_{1}(x)$ denotes the first kind of Bessel function of order one, and the argument $x$ relates to the radial distance, wavelength, and NA of the system.

The NA enhancement via SIL imaging becomes more evident when the image is compared to that of a conventional microscope (Fig. 3). The line profiles of the discs, which fit well with the Airy disc function, have a full-width at half-maximum (FWHM) of $245 \mathrm{~nm}$ and $565 \mathrm{~nm}$, with and without the SIL, respectively. From the fitting results, the effective NA is 0.54 for the conventional optical microscope,
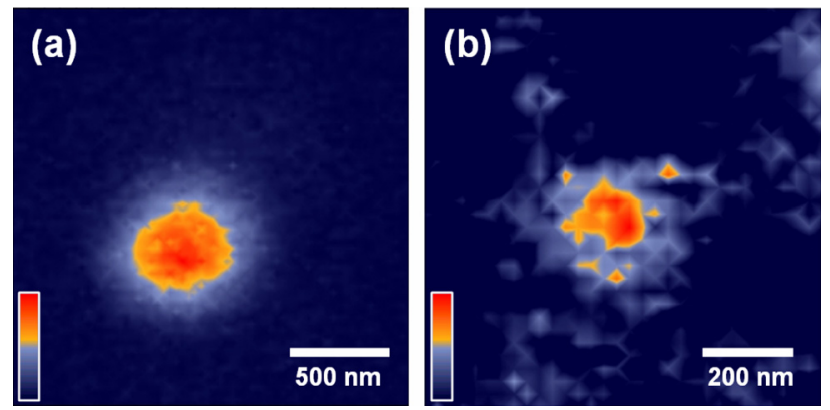

FIG. 4. Scanning mode PL images of single CdSe nanocrystals: (a) scanning optical microscope images and (b) scanning SIL microscope images.

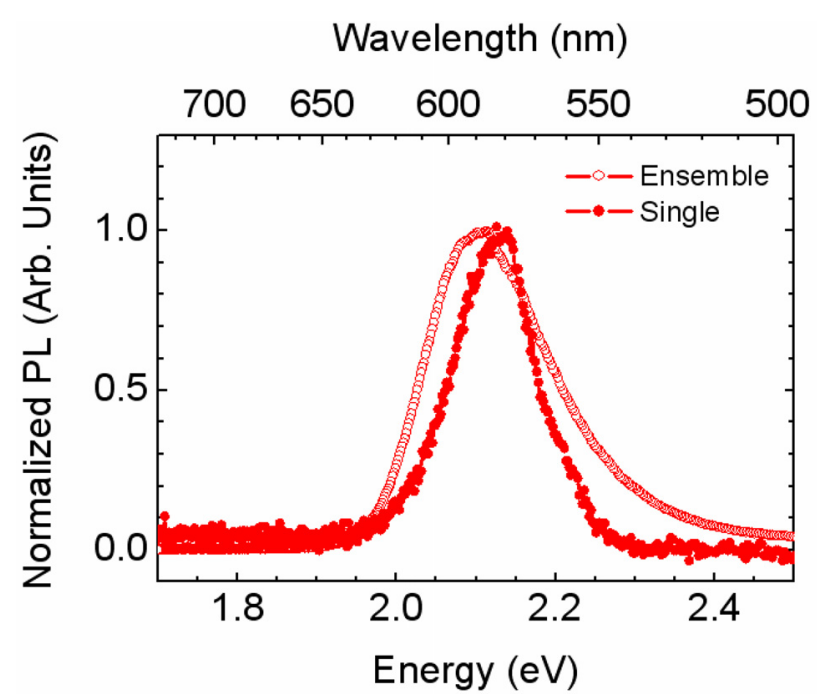

FIG. 5. PL spectrum of a single CdSe nanocrystal at room temperature. For comparison, the PL spectrum of CdSe nanocrystals in an ensemble state is added.

and 1.22 for the SIL microscope. These values agree well with the single-point resolution, i.e., the FWHM of a diffraction limited spot size: $\Delta x=0.51 \lambda_{e m} / N A$, where $\lambda_{e m}$ denotes the emission wavelength.

The PL images of scanning mode exhibit nearly same width as those of wide-field mode (Fig. 4). The fitting results show a FWHM of $569 \mathrm{~nm}$ for the scanning optical microscope, and $245 \mathrm{~nm}$ for the scanning SIL microscope, due to the fact that we employed extended illumination over the sample plane. Note that if the confocal condition is fulfilled, i.e., the excitation beam is focused to a diffractionlimited spot, and the emission signal is collected by a pinhole detector, the lateral resolution would be $\Delta x=0.37 \bar{\lambda} / N A$, where $\bar{\lambda} \cong \sqrt{\lambda_{\text {exc }} \cdot \lambda_{\text {em }}}$, and $\lambda_{\text {exc }}$ denotes the excitation wavelength. Calculations then show that an $\sim 150 \mathrm{~nm}$ lateral resolution should be realistic for scanning SIL imaging based on the given experimental conditions. In any case, the wide-field SIL microscope provides an affordable NA enhancement factor of 2 while maintaining most merits of a far-field optical microscope. 
Finally, note that we could measure the spectra of every single point in the PL images. A typical spectrum of a single CdSe nanocrystal at room temperature is shown in Fig. 5. The linewidth of PL signal is $118 \mathrm{meV}$, which is significantly narrower than that of the inhomogeneously broadened PL spectrum, due to size irregularities in the ensemble state. Subsequently, the SIL microscope offers a simple method for spectroscopy of nanostructures such as multiple quantum wells, quantum dots, nanowires, molecules, organic films, and bio samples.

\section{CONCLUSION}

In summary, we have successfully demonstrated a highspatial-resolution imaging and spectroscopy tool using a hemispherical SIL. Measured PL images of single CdSe nanocrystals confirmed an NA enhancement factor of $\sim 2$, close to the refractive index of the SIL. Notably, a bare-eye observation of PL signals emitted by single nanocrystals having $\sim 10 \mu \mathrm{m}^{-2}$ densities was possible over an $\sim 30 \mu \mathrm{m}$ diameter region. Furthermore, the PL spectrum from a single CdSe nanocrystal was successfully measured at room temperature using this instrument; thus, the SIL microscope ensures a simple but powerful method for nanostructure spectroscopy.

\section{ACKNOWLEDGMENT}

This research was supported by the Pioneer Research Center Program (2010-0002212), and by the Basic Science Research Program (2010-0012738) through the National
Research Foundation of Korea (NRF) funded by the Ministry of Education, Science and Technology, Korea. The authors also thank Dr. C.-L. Lee for his kind supplying of CdSe nanocrystal samples, and K.-D. Park for sample preparation.

\section{REFERENCES}

1. C. Sheppard and D. Shotton, Confocal Laser Scanning Microscopy (Springer, New York, USA, 1998).

2. L. Novotny and B. Hecht, Principles of Nano-optics (Cambridge University Press, Cambridge, UK, 2006).

3. S. W. Hell, "Far-field optical nanoscopy," in Single Molecule Spectroscopy in Chemistry, Physics and Biology, ed. (Springer, Berlin, Germany, 2009).

4. G. S. Kino, "Applications and theory of the solid immersion lens," Proc. SPIE 3609, 56-66 (1999).

5. S. M. Mansfield and G. S. Kino, "Solid immersion microscope," Appl. Phys. Lett. 57, 2615-2616 (1990).

6. B. D. Terris, H. J. Mamin, D. Rugar, W. R. Studenmund, and G. S. Kino, "Near-field optical data storage using a solid immersion lens," Appl. Phys. Lett. 65, 388-390 (1994).

7. Q. Wu, G. D. Feke, R. D. Grober, and L. P. Ghislain, "Realization of numerical aperture 2.0 using a gallium phosphide solid immersion lens," Appl. Phys. Lett. 75, 4064-4066 (1999).

8. M. Nirmal, B. O. Dabbousi, M. G. Bawendi, J. J. Macklin, J. K. Trautman, T. D. Harris, and L. E. Brus, "Fluorescence intermittency in single cadmium selenide nanocrystals," Nature 383, 802-804 (1996).

9. T. R. Corle and G. S. Kino, Confocal Scanning Optical Microscopy and Related Imaging Systems (Academic Press, San Diego, USA, 1996), Chapter 3. 